The universal equivariant genus and Krichever's formula

Buchstaber, V. M and Ray, N

2007

MIMS EPrint: 2007.101

Manchester Institute for Mathematical Sciences

School of Mathematics

The University of Manchester

\footnotetext{
Reports available from: http://eprints.maths.manchester.ac.uk/

And by contacting: The MIMS Secretary

School of Mathematics

The University of Manchester

Manchester, M13 9PL, UK
} 


\section{The universal equivariant genus and Krichever's formula}

\section{M. Buchstaber and N. Ray}

We consider smooth $2 n$-dimensional manifolds $M$, equipped with a smooth action $\theta$ of the circle $S^{1}$. Such an $M$ determines a normally complex $S^{1}$-manifold $\left(M, \theta, c_{\nu}\right)$ whenever an equivariant complex structure $c_{\nu}$ is chosen for the normal bundle $\nu(i)$ of some equivariant embedding $i: M \rightarrow \mathbb{C}^{k}$. In this situation, we use the standard action of $S^{1}$ on the unit sphere $S^{2 m+1} \subset \mathbb{C}^{m+1}$ to define a $2(m+n)$-dimensional smooth manifold $W_{m}=S^{2 m+1} \times_{S^{1}} M$, and a $k$-dimensional complex vector bundle $q_{m}: E_{m} \rightarrow \mathbb{C} P^{m}$, where $E_{m}=S^{2 m+1} \times_{S^{1}} \mathbb{C}^{k}$. Then $i$ extends to an embedding $i^{\prime}: W_{m} \rightarrow E_{m}$, and the complex structure $c_{\nu}$ extends to a complex structure $c^{\prime}$ on the normal bundle $\nu\left(i^{\prime}\right)$.

The composition $p_{m}: W_{m} \stackrel{i^{\prime}}{\longrightarrow} E_{m} \stackrel{q_{m}}{\longrightarrow} \mathbb{C} P^{m}$ is complex oriented in the sense of [1] and determines a complex cobordism class $\Phi_{m}\left(M, \theta, c_{\nu}\right) \in U^{-2 n}\left(\mathbb{C} P^{m}\right)$. The standard embedding $\iota_{m}: \mathbb{C} P^{m} \rightarrow \mathbb{C} P^{m+1}$ acts by $\iota_{m}^{*} \Phi_{m+1}=\Phi_{m}$, hence the inverse sequence $\left(\Phi_{m}\left(M, \theta, c_{\nu}\right): m \geqslant 0\right)$ defines an element of $\lim ^{-2 n}\left(\mathbb{C} P^{m}\right) \simeq U^{-2 n}\left(\mathbb{C} P^{\infty}\right)$. We write this element as $\Phi\left(M, \theta, c_{\nu}\right)$ and call it the universal $S^{1}$-equivariant genus of $\left(M, \theta, c_{\nu}\right)$. The genus $\Phi$ was used to deduce fundamental results on Hirzebruch genera of $S^{1}$-manifolds in [2] and [3]. An analogue of $\Phi$ for a compact Lie group has been studied by several authors since the 1960s (see [4] and [1]).

The ring $U^{*}\left(\mathbb{C} P^{\infty}\right)$ is isomorphic to the algebra $\Omega^{*}[[u]]$ of formal power series over $\Omega^{*}=U^{*}(p t)$, where $u$ is the cobordism class of the inverse sequence $\left(\iota_{m}: m \geqslant 0\right)$. So our initial aim is to construct stably complex manifolds $G_{j}(M)$ of dimension $2(n+j)$ such that $\Phi\left(M, \theta, c_{\nu}\right)=\sum_{j=0}^{\infty}\left[G_{j}(M)\right] u^{j}$. Clearly, $G_{0}=M$ with the stably complex structure $c_{\nu}$.

We consider $\left(S^{3}\right)^{j}=\left\{\left(y_{1}, z_{1} ; \ldots ; y_{j}, z_{j}\right) \in \mathbb{C}^{2 j}:\left|y_{i}\right|^{2}+\left|z_{i}\right|^{2}=1,1 \leqslant i \leqslant j\right\}$, on which the torus $T^{j}$ acts freely by $t \cdot\left(y_{1}, z_{1} ; \ldots ; y_{j}, z_{j}\right)=\left(t_{1} y_{1}, t_{1}^{-1} z_{1} ; \ldots ; t_{j} y_{j}, t_{j-1}^{-1} t_{j}^{-1} z_{j}\right)$ for all $t=\left(t_{1}, \ldots, t_{j}\right)$. The quotient manifold $B_{j}=\left(S^{3}\right)^{j} / T^{j}$ is a $j$-fold iterated $S^{2}$-sphere bundle over $B_{0}=p t$ and admits complex line bundles $\eta_{1}, \ldots, \eta_{j}$ such that $E\left(\eta_{i}\right)=\left(S^{3}\right)^{j} \times_{T^{j}} \mathbb{C}$ via the action $t \cdot z=t_{i}^{-1} z$ for $z \in \mathbb{C}$. For $j>0$, the isomorphism $\tau\left(B_{j}\right) \oplus \mathbb{C}^{j} \cong\left(\bar{\eta}_{1} \oplus \eta_{1}\right) \oplus$ $\cdots \oplus\left(\bar{\eta}_{j} \oplus \eta_{j-1} \eta_{j}\right)$ defines a stably complex structure $c_{j}^{\partial}$ such that $B_{j}=\partial D_{j}$, where $D_{j}$ is the associated 3 -disc bundle, hence $\left[B_{j}\right]=0$ for $j>0$.

Theorem 1. For every $j \geqslant 0$ the manifold $G_{j}(M)$ is given by the quotient $\left(S^{3}\right)^{j} \times_{T^{j}} M$, where $T^{j}$ acts on $M$ by $t \cdot x=\theta\left(t_{j}^{-1}\right) \cdot x$. The stably complex structure $c(j)$ on $G_{j}(M)$ is induced from the fibration $M \rightarrow G_{j}(M) \rightarrow B_{j}$.

The proof combines 1.7 in [1] with the fact (Proposition 2.2 in [5]) that the classifying maps for the bundles $\eta_{j}$ over $B_{j}$ represent a basis for $U_{*}\left(\mathbb{C} P^{\infty}\right)$ that is dual to the basis $\left\{u^{k}: k=0,1,2, \ldots\right\}$ in $U^{*}\left(\mathbb{C} P^{\infty}\right)$.

Remark 2. Our $G_{j}\left(M^{2 n}\right)$ are clearly complex bordant to the non-connected manifolds $\gamma^{j}\left(M^{2 n}\right)$ of Theorem 6.3 in [6]. Therefore, we deduce that the completion map of the homotopical bordism ring $M U_{*}^{S_{1}}$ at its augmentation ideal (see Ch. 27 in [7]) restricts to $\Phi$ on geometrical classes. Also, the $G_{j}\left(M^{2 n}\right)$ are diffeomorphic to the manifolds $M^{2(n+j)}$ of [8], so $c(j)$ induces a stably complex structure on the latter.

We interpret $M$ as a tangentially stably complex $S^{1}$-manifold $\left(M, \theta, c_{\tau}\right)$ whenever a complex vector bundle $\xi \rightarrow M$ and an isomorphism

$$
c_{\tau}: \tau(M) \oplus \mathbb{C}^{l-n} \rightarrow \xi
$$

AMS 2000 Mathematics Subject Classification. Primary 55N22; Secondary 57R85.

DOI 10.1070/RM2007v062n01ABEH004385. 
are chosen, where $\tau(M)$ is the stable tangent bundle, in such a way that the composition

$$
\rho(t): \xi \stackrel{c_{\tau}^{-1}}{\longrightarrow} \tau(M) \oplus \mathbb{C}^{l-n} \stackrel{d \theta(t) \oplus I}{\longrightarrow} \tau(M) \oplus \mathbb{C}^{l-n} \stackrel{c_{\tau}}{\longrightarrow} \xi, \quad t \in S^{1},
$$

is a complex transformation for any $t \in S^{1}$, where $d \theta(t)$ is the differential of the action by $\theta(t)$. Hence, $(2)$ corresponds to a representation $\rho: S^{1} \rightarrow \operatorname{Hom}_{\mathbb{C}}(\xi, \xi)$. Up to natural equivalence, the structure $\left(M, \theta, c_{\tau}\right)$ determines $\left(M, \theta, c_{\nu}\right)$, and we may define $\Phi\left(M, \theta, c_{\tau}\right)$ to be the $\Phi\left(M, \theta, c_{\nu}\right)$ associated with the corresponding structure $c_{\nu}$. On the other hand, $c_{\nu}$ may not determine an equivariant $c_{\tau}$ ([7], Ch. 28).

Let $x \in M$ be an isolated fixed point of the action $\theta$. Then the associated representation $\rho_{x}: S^{1} \rightarrow G L(l, \mathbb{C})$ decomposes the fibre $\xi_{x} \cong \mathbb{C}^{l}$ as $\mathbb{C}^{n} \oplus \mathbb{C}^{l-n}$, where $\rho_{x}$ acts (without trivial summands) as $\rho_{x, 1} \oplus \cdots \oplus \rho_{x, n}$ on $\mathbb{C}^{n}$ and trivially on $\mathbb{C}^{l-n}$. Here $\rho_{x, j}$ is the one-dimensional representation of weight $\omega_{x, j}$, that is, it acts by $\rho_{x, j}(t) v=t^{\omega_{x, j}} v$. To each isolated fixed point $x$ we may therefore assign a sequence $\omega_{x}=\left(\omega_{x, 1}, \ldots, \omega_{x, n}\right)$ of weights. Moreover, the isomorphism $c_{x, \tau}$ in (1) induces an orientation of the tangent space $\tau_{x}$, which allows us to define $\sigma(x)$ as the sign of the determinant of the linear map

$$
\tau_{x}(M) \stackrel{I \oplus 0}{\longrightarrow} \tau_{x}(M) \oplus \mathbb{C}^{l-n} \stackrel{c_{\tau, x}}{\longrightarrow} \xi_{x} \cong \mathbb{C}^{n} \oplus \mathbb{C}^{l-n} \stackrel{\pi}{\longrightarrow} \mathbb{C}^{n}
$$

where $\pi$ is the projection onto the first summand. The sign is important in toric topology [9].

Example 3. The $S^{1}$-manifold $\left(B_{1}=S^{2}, \theta, c_{1}^{\partial}\right)$, where $\theta(t)\left[z_{1}: z_{2}\right]=\left[t z_{1}: z_{2}\right]$, has fixed points $x_{1}=[1,0]$ and $x_{2}=[0,1]$ with weights $\omega_{x_{1}, 1}=\omega_{x_{2}, 1}=1$ and signs $\sigma\left(x_{1}\right)=$ $-\sigma\left(x_{2}\right)=1$.

Now let $\left\{[q](u) \in \Omega^{*}[[u]]: q \in \mathbb{Z}\right\}$ denote the power system of the formal group $f(u, v)$ for complex cobordism (see $[10])$. The $q$-series $[q](u)=q u \bmod \left(u^{2}\right)$ is defined uniquely by $[0](u)=0$ and $[q](u)=f(u,[q-1](u))$ for all $q \in \mathbb{Z}$.

Theorem 4. If the action $\theta$ has a finite set $X$ of isolated fixed points, then

$$
\Phi\left(M, \theta, c_{\tau}\right)=\sum_{x \in X} \sigma(x) \prod_{i=1}^{n} \frac{1}{\left[\omega_{x, i}\right](u)}
$$

holds in $U^{-2 n}\left(\mathbb{C} P^{\infty}\right)$.

The proof combines Proposition 3.8 in [1] with the construction of $\sigma(x)$ above.

Remark 5. If the $S^{1}$-manifold $M$ is almost complex, then we may take $l=n$ in (1). Then $\sigma(x)=1$ for all $x \in X$, and (3) reduces to Krichever's formula ((2.7) in [2]).

We may reformulate Theorems 1 and 4 for effective actions of the torus $T^{k}$ on any $2 n$-dimensional manifold with $k \leqslant n$.

\section{Bibliography}

[1] D. Quillen, Adv. Math. 7:1 (1971), 29-56.

[2] И. М. Кричевер, Изв. АН СССР. Сер. матем. 38:6 (1974), 1289-1304; English transl., I. M. Krichever, Math. USSR Izv. 8 (1974), 1271-1285.

[3] И. М. Кричевер, Матем. заметки 47:2 (1990), 34-45; English transl., I. M. Krichever, Math. Notes 47:2 (1990), 132-142.

[4] T. tom Dieck, Topology 9:4 (1970), 345-358.

[5] N. Ray, Proc. Edinb. Math. Soc. (2) 29:3 (1986), 413-422. 
[6] D. P. Sinha, Amer. J. Math. 123:4 (2001), 577-605.

[7] J. P. May (ed.), Equivariant homotopy and cohomology theory, CBMS Reg. Conf. Ser. Math., vol. 91, Amer. Math. Soc., Providence, RI 1996.

[8] P. E. Conner and E. E. Floyd, Differentiable periodic maps, Ergeb. Math. Grenzgeb., vol. 33, Springer-Verlag, Berlin-Göttingen-Heidelberg 1964.

[9] V. M. Buchstaber, T. E. Panov, and N. Ray, Mosc. Math. J. 7:2 (2007), 219-242.

[10] С. П. Новиков, Изв. АН СССР. Сер. матем. 31:4 (1967), 855-951; English transl., S. P. Novikov, Math. USSR Izv. 1:4 (1967), 827-913.

\section{M. Buchstaber}

Steklov Mathematical Institute, RAS

E-mail: buchstab@mi.ras.ru

\section{N. Ray}

School of Mathematics, University of Manchester,

England

E-mail: nige@ma.man.ac.uk
Presented by S. P. Novikov

Received 07/DEC/06

Translated by THE AUTHORS 